\title{
Editorial
}

\section{Violence and the night-time economy: A multi-professional perspective. An introduction to the Special Issue}

Crime Prevention and Community Safety (2009) 11, 147-152.

doi:10.1057/cpcs.2009.10

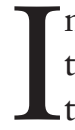

nternationally, a wealth of studies has demonstrated a link between the provision of alcohol and the occurrence of crime and disorder in the night-time economy (NTE), particularly in town and city centres (Olsson and Wikstrom, 1982; Rush et al, 1986; Edwards et al, 1994; Chikritzhs and Stockwell, 2002; Goodacre, 2005). However, the occurrence of 'alcohol-related' crime and disorder is a complex multidimensional issue, as the consumption of alcohol is not inevitably associated with involvement in violence or antisocial behaviour, and can only be addressed through an understanding of a diverse range of factors. This is an area that has been widely researched; yet, debate still exists as to how best to define and measure the extent of alcohol-related violence and disorder, how to identify causal factors, and, moreover, how best to tackle the problem.

To examine alcohol-related violence and disorder within the NTE, a range of contributory factors need to be examined. For example, to interpret what is happening requires an understanding on a very small scale and local level of the cultural and contextual factors associated with alcohol consumption. This may be within individual premises, around 'drinking circuits', or within particular areas with a number of licensing establishments in close proximity. There are also inherent methodological challenges in defining and measuring alcohol-related crime in the NTE. There is a need for better evidence, based on alcohol-related crime and disorder, which is both consistent and reliable. This is crucial for both a better understanding, locally and nationally, of the nature of the problem, and for selecting and targeting appropriate preventive measures within urban (and rural) areas. Moreover, alcohol-related crime, especially violence, has wider consequences for both personal health and the delivery of health services. These include both short-term consequences for accident and emergency units and the ambulance service, and the longer-term demands on Primary Care Trusts (PCTs) and the National Health Service (NHS) from prolonged and heavy alcohol consumption. The implementation of prevention and reduction policies on the ground 
needs to consider these broad-ranging factors. Therefore, it is essential to draw upon the experience of experts and professionals in different fields (for example, socio-legal, law enforcement, town and city planning, and public health) to both understand and address alcohol-related violence and disorder in the NTE.

In England and Wales, the Licensing Act 2003 (LA03) was introduced amidst growing concerns over problems of alcohol-related violence, especially in town and city centres in and around licensed premises. It represented the first major change to the sale of alcohol in England and Wales in 50 years. It brought with it a set of new conditions for the regulation and supply of alcohol, including the potential to make it available for up to 24 hours per day. The Act stimulated much media attention and political debate. It also resulted in a number of research studies within England and Wales regarding its impact. However, despite several national evaluations, doubt still exists as to the success of the Act, its impact on crime and disorder, and, more importantly, directions for future policy. Significantly, the evidence from Britain does not mirror experiences in other countries.

This Special Issue brings together perspectives and research evidence from the fields of sociology, criminology, and public health, as well as international comparisons and a contribution from practitioners to shed light on violence and disorder in the NTE. These papers bring together a range of experiences that reflect upon the impact of LA03 from alternative standpoints, and, more widely, to assess the effectiveness of policy responses beyond just the LA03. However, before introducing the papers brought together in this Special Issue, a brief description of the LA03 is first provided to set this in context for the reader.

\section{The LA03 in England and Wales}

The LA03 came into effect on 24 November 2005, and this constituted a major revision to the sale of alcohol in England and Wales, potentially allowing licensed premises to sell alcohol for up to 24 hours, 7 days per week. The rationale behind the Act was that by removing fixed and artificially early closing times, the numbers of people exiting licensed premises would be dispersed over a longer time period. The expectation was that this would reduce binge drinking, violent behaviour, and damage to property and disorder. It was also designed to make it easier for authorities to deal with problem premises, and to complement other measures within the criminal justice system designed to crackdown on consumers and providers of alcohol who go beyond the boundaries of what is lawful or socially acceptable. At the same time, concerns were voiced that the Act would lead to greater alcohol consumption, increased levels of violence, and more pressure on accident and emergency units. The 
Act brought with it a set of new conditions and additional powers, and signalled a major change to the licensing laws in England and Wales. Some of the key measures summarized by the Act are now described below (DCMS, 2009).

- Flexible opening hours for premises, with the potential for up to 24 hours opening, seven days a week, subject to consideration of the impact on local residents, businesses, and the expert opinion of a range of agencies, which were designated as responsible authorities by the Act, in relation to the licensing objectives.

- The Act required each licensing authority to carry out its duties with a view to promoting the four main licensing objectives:

1. The prevention of crime and disorder;

2. Public safety;

3. The prevention of public nuisance; and

4. The protection of children from harm.

- A single, integrated scheme for licensing premises, which sell alcohol, provide public entertainment or provide refreshment late at night based upon a 'premises license'. This brought together the existing regimes, and saw the end of the current system based upon 'public entertainment licenses' (for premises that provided entertainment to the public); 'late night refreshment licenses' (for premises that were open for refreshment and entertainment between 2200 and 0500 hours); and 'special hours certificates' (for premises staying open later than normal licensing hours). These were ancillary to 'public entertainment licenses'.

- A personal license system required by licensees, which will facilitate greater mobility for holders to move between premises, possessing a premises license.

There were a range of key changes. The responsibility for licensing decisions shifted from the local magistrates' courts to local governments (local authorities), who effectively became the new licensing authorities. This gave local authorities more control and flexibility over licensing in their area. Additionally, responsible authorities could now request one or more specific conditions to be placed on an individual premise for a license to be granted. However, the onus was on the authority to prove that without the prescribed condition(s), a premise would contravene one of the four licensing objectives stated above. Responsible authorities could include any one or more of the following: licensing authorities, environmental health, trading standards, crime and disorder reduction partnerships (CDRPs), planning, town centre management, the drugs and alcohol team, the fire service, and police licensing 
officers. Examples of conditions that could be imposed include installation of CCTV cameras, a limit on capacity, serving of food, or a particular number of door supervisors to work on certain nights of the week.

The Act gave powers to local authorities to introduce cumulative impact (saturation) polices. If they could prove that an area was already saturated with licensed premises, they were given the ability to prevent the granting of licenses to any new premises. However, if an individual premise could prove that it would not add to crime and disorder in an area, it could successfully apply for a license. Difficulties in establishing an evidence base for this will be discussed in one of the papers in this Special Issue. Police powers were also enhanced by the Act, including closure of premises for breaching its licensing conditions, and confiscation of alcohol to prevent consumption in public places, targeting young people through the Confiscation of Alcohol (Young Persons) Act 1997, and particular places (through designated public place orders (DPPOs), under the Criminal Justice and Police Act 2001.

\section{Violence and the NTE: A multiprofessional perspective}

This Special Issue offers five papers that discuss the complexities of violence and the NTE, and issues around extended trading hours of licensed premises, from different specialists' viewpoints. These include contributions from sociological, criminological, public health, and practitioner perspectives.

The first paper, by Tim Stockwell and Tanya Chikritzhs, reports the findings from a review of international research carried out by the authors, setting out the use of extended licensing hours in an international setting. They use a systematic literature search to identify studies that have attempted to evaluate the public health and safety impacts of changes to the trading hours of outlets on premise alcohol consumption. A total of 49 unique studies met the inclusion criteria. These were divided into three categories based on methodological rigour. The paper concludes that differences found between UK and other studies are most likely due to differences in methodological approaches. They suggest that based on international evidence as a whole, the likelihood is that extending trading hours for licensed premises leads to increased consumption and related harms (beyond just violence), and that more well-controlled studies are required to confirm this conclusion.

The second paper, by Andrew Newton and Alex Hirschfield, examines the state of the current evidence base for those faced with policing and managing the NTE. It uses analysis and findings from the author and colleagues' evaluation of LA03 (Newton et al, 2008) to show both the limitations of current intelligence and what can be achieved by using just two sources of data, namely, police violence against the person (VAP) and information on outlets that supply alcohol to the public (alcohol supply points). It highlights the 
importance of 'high risk' premises and areas with a concentration of licensed premises, the importance of time (both time of day and day of week), and the value of local contextual knowledge (for example, the difference between the licensed trading hours granted and those actually used) in measuring and interpreting change. It identifies some additional sources of data that might be used to improve the current level of intelligence on the nature and extent of alcohol-related violence in the NTE, and discusses some of the potential obstacles and drawbacks in using these. It concludes by highlighting the necessity for a reliable and consistent evidence base for managing and policing the NTE.

The third paper, by Amy Burrell and Rosie Erol, discusses the effects of key policy changes in England and Wales, including the introduction of extended licensing hours under the LA03 from a practitioner's perspective, examining the day-to-day delivery of frontline services. The paper highlights how national crime reduction initiatives and strategies, such as the tackling violent crime programme (TVCP) and alcohol misuse enforcement campaigns (AMECs), were implemented on a local level. Local interventions, such as the use of head-mounted cameras for evidence gathering and the introduction of Best Bar None schemes, are also discussed in relation to NTE violence. It uses a case study of an anonymous town centre to examine decisions faced by practitioners tasked with managing the NTE at a local level.

The fourth paper, by Michela Morleo and colleagues, examines violence and the NTE from a public health perspective. It examines the impact of LA03 on levels of violence, on alcohol consumption, and on children. It then advocates the need to consider licensing from a public health perspective and the need for this to be incorporated into decisions about the granting and renewal of licenses. The paper draws extensively upon international experiences of violence and the NTE from a public health perspective. It identifies a range of challenges faced in implementing LA03 and reports mixed messages about its impact on violence. It concludes by arguing that the consideration of alcoholrelated violence as a public health issue is intrinsic to being effective in tackling violence in nightlife settings.

Finally, the fifth paper, by Phil Hadfield and Fiona Measham, considers the impact of the LA03 within the broader sociological and cultural context of the NTE. It discusses how the LA03 provides a framework that shapes the content of the NTE, effectively regulating different forms of social activity, endorsing some and suppressing others. This is explained through a detailed examination of 'lap-dancing' venues and premises offering live music. The paper then examines those who participated in the NTE looks at the homogeneity of such groups and argues in favour of the diversity of experiences of those who participated in the NTE, demonstrating this through the use of detailed case field notes. It argues that the available evidence suggests that LA03 does not appear to have transformed the drinking culture of England and Wales. 
This Special Issue concludes with two book reviews that are highly relevant to the discussion of violence and the NTE. Kathryn Graham and Ross Homel's book, Raising the Bar: Preventing Aggression in and around Bars, Pubs and Clubs, is reviewed by Melanie Wellsmith. David Humphreys also reviews Phil Hadfield's edited collection entitled Nightlife and Crime: Social Order and Governance in International Perspective.

\section{References}

Chikritzhs, T. and Stockwell, T. (2002) The impact of later trading hours for hotels on levels of violence. Journal of Studies on Alcohol 63(5): 591-599.

Department for Culture, Media and Sport (DCMS). (2009) Licensing Act 2003 Explained, http://www.culture.gov.uk/what_we_do/alcohol_and_entertainment/4051. aspx, accessed 21 February 2007.

Edwards, G. et al (1994) Alcohol Policy and the Public Good. Oxford: Oxford University Press.

Goodacre, S. (2005) The 2003 licensing act: An act of stupidity? Emergency Medical Journal 22(10): 682 .

Newton, A., Hirschfield, A., Armitage, R., Rogerson, M., Monchuk, L. and Wilcox, A. (2008) Evaluation of the licensing act: Measuring crime and disorder in and around licensed premises. Research Study SRG/05/07. Final Report Prepared for the Home Office. Huddersfield: University of Huddersfield.

Olsson, O. and Wikstrom, P. (1982) Effects of experimental Saturday closing of liquor retail stores in Sweden. Contemporary Drug Problems 11: 325-353.

Rush, B.R., Glicksman, L. and Brook, R. (1986) Alcohol availability, alcohol consumption and alcohol related damage: The distribution of consumption model. Journal of Studies on Alcohol 47: 1-10.

Andrew Newton

The Applied Criminology Centre (ACC), University of Huddersfield, Queensgate, Huddersfield, UK E-mail: a.d.newton@hud.ac.uk

Alex Hirschfield The Applied Criminology Centre (ACC), University of Huddersfield, Queensgate, Huddersfield, UK 